\title{
Predicting Mortality and Adverse Outcomes: Comparing the Frailty Index to General Prognostic Indices
}

\author{
Sandra M. Shi, MD ${ }^{1,2}$, Ellen P. McCarthy, $P h D^{1,2}$, Susan L. Mitchell, MD ${ }^{1,2}$, and \\ Dae Hyun Kim, MD ${ }^{1,2}$
}

\begin{abstract}
${ }^{1}$ Hinda and Arthur Marcus Institute for Aging, Hebrew Senior Life, Boston, MA, USA; ${ }^{2}$ Department of Medicine, Beth Israel Deaconess Medical Center, Harvard Medical School, Boston, MA, USA.
\end{abstract}

BACKGROUND: Mortality prediction models are useful to guide clinical decision-making based on prognosis. The frailty index, which allows prognostication and personalized care planning, has not been directly compared with validated prognostic models.

OBJECTIVE: To compare the discrimination of mortality, disability, falls, and hospitalization between a frailty index and validated prognostic indices.

DESIGN: Secondary Analysis of the National Health and Aging Trends Study.

PARTICIPANTS: Seven thousand thirty-three Medicare beneficiaries 65 years or older.

MEASUREMENTS: We measured a deficit-accumulation frailty index, Schonberg index, and Lee index at the 2011 baseline assessment. Primary outcome was mortality at 5 years. Secondary outcomes were decline in activities of daily living (ADL), decline in instrumental activities of daily living (IADL), fall, and hospitalization at 1 year. We used C-statistics to compare discrimination between indices, adjusting for age and sex.

RESULTS: The study population included 4146 (44.8\%) with age $\geq 75$ years, with a median frailty index of 0.15 (interquartile range 0.09-0.25). A total of 1385 participants died (14.7\%) and 2386 (35.2\%) were lost to follow-up. Frailty, Schonberg, and Lee indices predicted mortality similarly: C-statistics (95\% confidence interval) were 0.78 (0.77-0.80) for frailty index; $0.79(0.78-0.81)$ for Schonberg index; and $0.78(0.77-0.80)$ for Lee index. The frailty index had higher C-statistics for decline in ADL function (frailty index, 0.80 [0.78-0.83]; Schonberg, 0.74 [0.72-0.76]; Lee, 0.74 [0.71$0.77]$ ) and falls (frailty index, 0.66 [0.65-0.68]; Schonberg, 0.61 [0.58-0.63]; Lee, 0.61 [0.59-0.63]). C-statistics were similar for decline in IADL function (frailty index, 0.61 [0.59-0.63]; Schonberg, 0.60 [0.59-0.62]; Lee, 0.60 [0.580.62]) and hospitalizations (frailty index, 0.68 [0.66-0.70]; Schonberg, 0.68 [0.66-0.69]; Lee, 0.65 [0.63-0.67]).

CONCLUSIONS: A deficit-accumulation frailty index performs as well as prognostic indices for mortality prediction, and better predicts ADL disability and falls in

Meeting Presentations An earlier version of this work was presented as a poster at the 2019 American Geriatrics Society annual meeting in Portland. Oreaon in Mau 2019.

Electronic supplementary material The online version of this article (https://doi.org/10.1007/s11606-020-05700-w) contains supplementary material which is available to authorized users.

Received July 11, 2019

Accepted February 3, 2020

Published online February 18, 2020 community-dwelling older adults. Frailty assessment offers a unifying approach to risk stratification for key health outcomes relevant to older adults.

KEY WORDS: frailty; prognostication; mortality; disability; prediction

J Gen Intern Med 35(5): 1516-22

DOI: $10.1007 / \mathrm{s} 11606-020-05700-w$

(C) Society of General Internal Medicine 2020

\section{INTRODUCTION}

Risk prediction is paramount to aid clinical decision-making and the delivery of care centered on individual goals and preferences in older adults. ${ }^{1-3}$ Estimating life expectancy or prognosis is a common approach to risk profiling, for example, deciding whether to pursue cancer screening. However, mortality risk may not reflect overall health status or risk of other health outcomes relevant to older adults, such as disability and independence. ${ }^{4}$ Applying a different prediction tool for each outcome of interest can be impractical, as multiple outcomes may be relevant to a single clinical decision. Furthermore, common prognostic models for mortality, such as the Schonberg index ${ }^{5}$ and Lee index, ${ }^{6}$ are based largely on demographic characteristics, diagnoses, and a limited range of selfreported functional status, and do not account for physical performance and cognitive function.

Frailty is a state of reduced physiologic reserve associated with aging. ${ }^{7,8}$ The deficit-accumulation model quantifies frailty by measuring age-associated cumulative deficits. ${ }^{9}$ By calculating the proportion of deficits present, a frailty index can identify older adults at increased risk of adverse outcomes, such as disability, hospitalizations, and mortality. ${ }^{10}$ Its comprehensiveness also allows identification of target domains for personalized interventions. Although the original frailty index was developed with 90 variables, subsequent work has demonstrated that the number of variables can be reduced to a more manageable 30. ${ }^{11}$ The frailty index has been applied in populations in the USA, ${ }^{12}$ Canada, ${ }^{10}$ and Europe, ${ }^{13}$ as well as in the context of peri-operative risk stratification. ${ }^{14}$ Although frailty is known to be associated with mortality, the performance of a frailty index has not been directly compared with prognostic indices. Because frailty accounts for a person's health status and overall complexity, it may be a more 
generalizable framework to approach weighing risks and benefits of interventions in patients with competing priorities.

In this study, we compare the prediction of mortality between a frailty index and validated prognostic indices in a nationally representative sample of community-dwelling older adults in the USA. In particular, we examined the predictive performance in population subgroups and for prediction of disability, falls, and hospitalizations.

\section{METHODS}

\section{Study Population}

The National Health and Aging Trends Study (NHATS) is a nationally representative sample of Medicare beneficiaries ages 65 and older, aimed to study national-level and individual trajectories of disability, sponsored by the National Institute on Aging (grant number NIA U01AG032947) through a cooperative agreement with the Johns Hopkins Bloomberg School of Public Health. ${ }^{15}$ Medicare is a US health insurance program for all persons 65 years and older, or people with certain disabilities. This study used data from the original 2011 (Round 1) cohort, followed through 2016. Trained research staffs conduct in-person interviews in the homes of participants annually, along with a proxy or caregiver if available. Informed consent was obtained from all study participants or their proxies. The Hebrew SeniorLife Institutional Review Board approved this study.

We included Medicare beneficiaries who participated in the Round 1 assessment $(n=8245)$. Participants were excluded if they were living in a nursing home $(n=468)$, were living in residential care $(n=580)$, did not have any survival data $(n=$ $24)$, or did not have sufficient information to calculate a frailty index ( $<35$ of 41 variables measured as described below, $n=$ 140). The final sample included 7033 participants.

\section{Measurements of Frailty and Prognostic Indices}

Using baseline information, we calculated a frailty index (Supplementary Table 1) based on items in a comprehensive geriatric assessment, the Schonberg index ${ }^{5}$ and Lee index. ${ }^{6}$ The frailty index included 41 variables: ${ }^{16} 13$ self-reported medical history items, 6 activities of daily living (ADLs - feeding, dressing, grooming, bed mobility, bathing, toileting), 7 instrumental activities of daily living (IADLs - using the telephone, transportation, shopping, meal preparation, housework, medication management, managing finances), 7 self-reported physical tasks (pushing/pulling heavy objects, stooping or kneeling, lifting $10 \mathrm{lbs}$., reaching arms above shoulder, handling small objects, walking up a flight of stairs, walking half a mile, heavy housework), 3 performance measures (gait speed, grip strength, chair stands), 3 neuropsychological measures (cognitive testing, Patient Health Questionnare-2, Generalized Anxiety Disorder-2), and 2 nutritional measures (body mass index [BMI] $<21 \mathrm{~kg}$ / $\mathrm{m}^{2}$, unintentional weight loss $>10 \mathrm{lbs}$.). The frailty index was calculated according to the deficit-accumulation model as the total number of deficits present, divided by the total number of potential deficits considered (range $0-1$ ). For example, if a participant had 10 deficits out of a total of 40 measured, the frailty index is $0.25(10 / 40)$.

The Schonberg index (range 0-29) is based on 11 weighted items: age, sex, BMI $<25 \mathrm{~kg} / \mathrm{m}^{2}$, self-reported health, lung disease, cancer, diabetes, smoking (never, former, current), hospitalizations in the past year (none, $1, \geq 2$ ), needing any help with IADLs, and ability to walk 3 blocks. The Lee index (range 0-26) is based on 12 weighted items: age, sex, BMI < $25 \mathrm{~kg} / \mathrm{m}^{2}$, lung disease, cancer, diabetes, congestive heart failure, current smoking, difficulty bathing, difficulty with finances, difficulty pushing/pulling large objects, and difficulty walking several blocks. Based on the total score, a risk of mortality can be calculated for 4 (Lee index) or 5 years (Schonberg index). Higher scores reflect a higher risk of mortality and thus poorer prognosis.

We constructed 5 risk categories for each index, based on percentile distribution in the overall study population $(<10$ th, 10-24th, 25-74th, 75-89th, and $\geq 90$ th percentile).

\section{Baseline Measurements}

Age is reported categorically $(65-69,70-71,75-79,80-84$, $85-89$, and $\geq 90$ years). Sex, race, medical comorbidities (including dementia), height, and weight were self-reported by patient or proxy. Depression and anxiety were measured using PHQ-2 (score $\geq 2$ ) and GAD 2-item (score $\geq 2$ ), respectively. ${ }^{17}$ We defined hearing impairment and visual impairment based on previously used definitions in NHATS. ${ }^{18}$ Gait speed and grip strength were calculated as the average of two attempts. Time needed to complete 5 chair stands on a single attempt was measured. Cognitive impairment was defined as performance 1.5 standard deviations below the population mean in domains of orientation (date, month, year, day of the week, naming the President and Vice President), memory (immediate and delayed 10-word recall), or executive function (clock drawing test). ${ }^{19}$ Mobility impairment was defined as self-reported inability to climb a flight of stairs or walk 6 blocks.

\section{Outcomes}

The primary outcome was all-cause mortality. NHATS obtains month and year of death from follow-up interviews with caregivers. Observations were censored at the time of last known follow-up interview or 5 years from the baseline interview, whichever came first.

We examined the following secondary outcomes at 1-year follow-up interview among participants who were alive with complete follow-up interview $(n=5626)$. Decline in ADL function was defined as requiring assistance in more ADLs (out of 6 possible) compared with baseline. Decline in IADL function was defined similarly, using the 5 IADLs ascertained 
on follow-up interview (laundry, driving, shopping, meal preparation, and managing finances). Subjects with maximal ADL disability or IADL disability at baseline were excluded from respective analyses. Falls and hospitalizations were selfreported.

\section{Statistical Analysis}

We examined baseline demographics, comorbidities, and distribution of scores for all three indices in the overall cohort. The restricted mean survival time (RMST) is the average survival time in a pre-specified period (effectively the area under a Kaplan-Meier curve), providing more intuitive interpretation than probabilities. ${ }^{20}$ We calculated 5-year mortality from Kaplan-Meier curves and 5-year RMST for risk categories according to each index. Since the Schonberg index and Lee index include age and sex, while frailty index does not, we adjusted for age and sex in Cox proportional hazards models to estimate the independent association of each index with mortality. We used the somersd Stata package to calculate Cstatistics and $95 \%$ confidence intervals (CIs) for each model. ${ }^{21}$ We further examined the performance of each index within pre-specified subgroups defined by sex, age $(<75$ or $\geq 75$ years old), cognitive impairment, and mobility impairment.

We calculated the 1-year risk of decline in ADL function, IADL function, any fall, and any hospitalization for risk categories of each index. Logistic regression was used to assess the independent association of each index with these outcomes, adjusting for age and sex. C-statistics and 95\% CIs were estimated for each model. We repeated these analyses for each index in the aforementioned subgroups. All analyses were performed using Stata survey procedures (version 15.1, StataCorp, Texas) to account for the complex sampling design of NHATS, and findings were weighted to reflect national estimates in the Medicare population.

\section{RESULTS}

\section{Baseline Characteristics}

The study population included $4146(44.8 \%)$ with age $\geq$ 75 years, $4056(55.8 \%)$ women, and $2224(18.9 \%)$ nonwhite race (Table 1). At baseline, 997 (10.4\%) had ADL disability and 2327 (26.0\%) had IADL disability. The most prevalent comorbidity was hypertension $(n=4738,63.9 \%)$, followed by vision impairment $(n=4334,61.5 \%)$, whereas stroke $(n=806,9.7 \%)$ and dementia $(n=383,3.7 \%)$ were least common. Cognitive impairment was present in 1535 participants $(17.1 \%)$, and mobility impairment was present in 2866 participants $(33.5 \%)$.

The median frailty index score was 0.15 (interquartile range [IQR] 0.09-0.25). The median Schonberg index score was 7 (IQR 5-11), and median Lee index score was 7 (IQR 5-10). The 10th, 25th, 50th, 75th, and 90th percentile values for indices are as follows: $0.06,0.09,0.15,0.25$, and 0.40 for
Table 1 Baseline Characteristics of the Total Cohort $(n=7033)$

\begin{tabular}{|c|c|}
\hline Characteristic & $n(\%)^{*}$ \\
\hline \multicolumn{2}{|l|}{ Age } \\
\hline $65-69$ & $1374(29.5)$ \\
\hline $70-74$ & $1513(25.7)$ \\
\hline $75-79$ & $1422(19.3)$ \\
\hline $80-84$ & $1387(14.3)$ \\
\hline $85-89$ & $829(8.0)$ \\
\hline $90+$ & $508(3.2)$ \\
\hline Male & 2977 (44.2) \\
\hline \multirow{2}{*}{\multicolumn{2}{|c|}{ Race/ethnicity }} \\
\hline & \\
\hline Non-Hispanic White & $4809(81.1)$ \\
\hline Non-Hispanic Black & $1572(8.3)$ \\
\hline Hispanic & $436(7.0)$ \\
\hline Other $^{\dagger}$ & $216(3.6)$ \\
\hline \multicolumn{2}{|l|}{ Self-report comorbid conditions } \\
\hline Hypertension & $4738(63.9)$ \\
\hline Vision impairment & $4334(61.5)$ \\
\hline Arthritis & $3903(53.4)$ \\
\hline Spine disease & $2835(40.7)$ \\
\hline Diabetes & $1776(23.6)$ \\
\hline Heart disease & $1288(17.2)$ \\
\hline Cancer & $1196(16.2)$ \\
\hline Lung disease & $1076(15.5)$ \\
\hline Heart attack & $1055(13.7)$ \\
\hline Hearing impairment & $890(11.4)$ \\
\hline Stroke & $806(9.7)$ \\
\hline Dementia & $383(3.7)$ \\
\hline Any ADL disability & $997(10.4)$ \\
\hline Any IADL disability & $2327(26.0)$ \\
\hline Any fall in past year & $2170(29.8)$ \\
\hline Any hospitalization in past year & $1595(20.2)$ \\
\hline Abnormal cognitive testing & $1535(17.1)$ \\
\hline Mobility impairment & $2866(33.5)$ \\
\hline Screened positive for depression (PHQ-2 score $\geq 3$ ) & $1093(14.1)$ \\
\hline Screened positive for anxiety (GAD-2 score $\geq 3$ ) & $913(12.0)$ \\
\hline Median frailty index (IQR) & $0.15[0.09-0.25]$ \\
\hline Median Schonberg Score (IQR) & $7[5-11]$ \\
\hline Median Lee Score (IQR) & $7[5-10]$ \\
\hline
\end{tabular}

$*_{n}$ indicates sample size; percentages are weighted to reflect national estimates

†Other category includes American Indian, Alaskan Native, Asian, and unknown

Abbreviations: $A D L$, activities of daily living; $G A D$, general anxiety disorder 2-item; IADL, instrumental activities of daily living; $P H Q-2$, Patient Health Questionnaire-2; IQR, interquartile range

For frailty index (range 0-1), Schonberg index (range 0-29), and Lee index (range 0-26) higher scores reflect poorer prognosis. Cognitive impairment defined by abnormal performance in any of orientation, memory, executive function. Mobility impairment defined by selfreported inability to walk 6 blocks or climb a flight of stairs

frailty index; 3, 5, 7, 11, and 14 for Schonberg index; 4, 5, 7, 10 , and 13 for Lee index. Details regarding score distribution by age category and sex are in Supplemental Table 2.

\section{Prediction of Mortality at 5 Years}

Among 7033 participants, 1385 died (14.7\%) and 2386 (35.2\%) participants were lost to follow-up over 5 years (Table 2), with mean follow-up time was 43.5 months (26.0 months among those lost to follow-up). Baseline frailty, Schonberg, and Lee index scores were lower among those lost to follow-up, indicated better health (Supplementary Table 3).

Overall, higher risk categories had shorter 60-month RMST (40.8, 39.9, and 38.9 months for $\geq 90$ th percentile frailty, Schonberg, and Lee indices, respectively) (Fig. 1a) and higher 5 -year mortality risk $(56.4 \%, 61.0 \%$, and $62.3 \%$ for $\geq 90$ th 
Table 2 Comparison of Model C-Statistics [95\% Confidence Interval] for 5-Year Mortality and 1-Year Incidence of Adverse Outcomes

\begin{tabular}{|c|c|c|c|c|c|}
\hline & \multirow[t]{2}{*}{ 5-year mortality } & \multicolumn{4}{|l|}{ 1-year incidence } \\
\hline & & $\begin{array}{l}\text { Decline in ADL } \\
\text { function }\end{array}$ & $\begin{array}{l}\text { Decline in IADL } \\
\text { function }\end{array}$ & Fall & Hospitalization \\
\hline Total $n / N(\%)$ & $\begin{array}{l}1385 / 7033 \\
(14.7 \%)\end{array}$ & $747 / 5547(10.5 \%)$ & $1402 / 5224(24.6 \%)$ & $\begin{array}{l}1881 / 5626 \\
(32.6 \%)\end{array}$ & $\begin{array}{l}1397 / 5626 \\
(22.5 \%)\end{array}$ \\
\hline Frailty & $0.78[0.77-0.80]$ & $0.80[0.78-0.83]$ & $0.61[0.59-0.63]$ & $0.66[0.65-0.68]$ & $0.68[0.66-0.70]$ \\
\hline Schonberg & $0.79[0.78-0.81]$ & $0.74[0.72-0.76]$ & $0.60[0.59-0.62]$ & $0.61[0.58-0.63]$ & $0.68[0.66-0.69]$ \\
\hline Lee & $0.78[0.77-0.80]$ & $0.74[0.71-0.77]$ & $0.60[0.58-0.62]$ & $0.61[0.59-0.63]$ & $0.65[0.63-0.67]$ \\
\hline Age $\geq 75 n / N(\%)$ & $\begin{array}{l}1150 / 4146 \\
(23.9 \%)\end{array}$ & $571 / 3210(15.5 \%)$ & $870 / 2956(27.5 \%)$ & $\begin{array}{l}1203 / 3281 \\
(36.9 \%)\end{array}$ & $920 / 3281(27.2 \%)$ \\
\hline Frailty & $0.74[0.73-0.76]$ & $0.79[0.77-0.82]$ & $0.62[0.59-0.66]$ & $0.65[0.63-0.67]$ & $0.64[0.61-0.66]$ \\
\hline Schonberg & $0.75[0.73-0.77]$ & $0.71[0.69-0.74]$ & $0.61[0.60-0.63]$ & $0.59[0.57-0.62]$ & $0.62[0.60-0.65]$ \\
\hline Lee & $0.74[0.73-0.76]$ & $0.73[0.70-0.76]$ & $0.61[0.59-0.64]$ & $0.61[0.59-0.63]$ & $0.61[0.59-0.63]$ \\
\hline Age < $75 n / N(\%)$ & $235 / 2887(7.3 \%)$ & $176 / 2337(6.7 \%)$ & $532 / 2268(22.5 \%)$ & $678 / 2345(29.2 \%)$ & $477 / 2345(18.8 \%)$ \\
\hline Frailty & $0.75[0.71-0.78]$ & $0.78[0.73-0.82]$ & $0.59[0.56-0.62]$ & $0.66[0.63-0.69]$ & $0.70[0.67-0.73]$ \\
\hline Schonberg & $0.75[0.72-0.79]$ & $0.71[0.66-0.76]$ & $0.58[0.56-0.61]$ & $0.60[0.56-0.63]$ & $0.69[0.66-0.71]$ \\
\hline Lee & $0.73[0.70-0.77]$ & $0.69[0.64-0.75]$ & $0.58[0.55-0.60]$ & $0.59[0.56-0.62]$ & $0.64[0.61-0.67]$ \\
\hline Male $n / N(\%)$ & $633 / 2977(15.9 \%)$ & $259 / 2355(9.3 \%)$ & $511 / 2215(20.4 \%)$ & $739 / 2377(29.6 \%)$ & $590 / 2377(22.3 \%)$ \\
\hline Frailty & $0.77[0.74-0.80]$ & $0.80[0.76-0.84]$ & $0.60[0.56-0.63]$ & $0.68[0.65-0.71]$ & $0.68[0.65-0.71]$ \\
\hline Schonberg & $0.79[0.77-0.81]$ & $0.74[0.70-0.78]$ & $0.60[0.56-0.63]$ & $0.60[0.57-0.63]$ & $0.69[0.66-0.72]$ \\
\hline Lee & $0.78[0.75-0.80]$ & $0.73[0.69-0.77]$ & $0.60[0.56-0.64]$ & $0.61[0.58-0.64]$ & $0.65[0.63-0.68]$ \\
\hline Female $n / N(\%)$ & $752 / 4056(13.8 \%)$ & $488 / 3192(11.5 \%)$ & $890 / 3009(28.0 \%)$ & $\begin{array}{l}1142 / 3249 \\
(35.0 \%)\end{array}$ & $807 / 3249(22.6 \%)$ \\
\hline Frailty & $0.79[0.77-0.81]$ & $0.80[0.78-0.83]$ & $0.58[0.56-0.61]$ & $0.64[0.62-0.67]$ & $0.67[0.65-0.70]$ \\
\hline Schonberg & $0.79[0.77-0.81]$ & $0.74[0.71-0.77]$ & $0.57[0.54-0.59]$ & $0.59[0.57-0.62]$ & $0.66[0.64-0.69]$ \\
\hline Lee & $0.79[0.77-0.80]$ & $0.75[0.71-0.78]$ & $0.56[0.53-0.58]$ & $0.59[0.57-0.62]$ & $0.64[0.61-0.67]$ \\
\hline $\begin{array}{l}\text { Cognitive impairment } n / N \\
(\%)\end{array}$ & $538 / 1535(29.2 \%)$ & $298 / 1103(23.4 \%)$ & $329 / 931(34.4 \%)$ & $442 / 1140(38.1 \%)$ & $363 / 1140(29.6 \%)$ \\
\hline Frailty & $0.73[0.71-0.75]$ & $0.80[0.77-0.83]$ & $0.59[0.55-0.64]$ & $0.72[0.69-0.76]$ & $0.68[0.64-0.72]$ \\
\hline Schonberg & $0.73[0.70-0.75]$ & $0.74[0.71-0.77]$ & $0.59[0.55-0.63]$ & $0.66[0.62-0.70]$ & $0.67[0.63-0.71]$ \\
\hline Lee & $0.73[0.70-0.75]$ & $0.75[0.72-0.79]$ & $0.59[0.55-0.63]$ & $0.66[0.63-0.70]$ & $0.64[0.60-0.68]$ \\
\hline $\begin{array}{l}\text { Mobility impairment } n / N \\
(\%)\end{array}$ & $934 / 2866(27.9 \%)$ & $571 / 2138(23.7 \%)$ & $646 / 1856(32.8 \%)$ & $992 / 2205(46.4 \%)$ & $782 / 2205(34.7 \%)$ \\
\hline Frailty & $0.72[0.70-0.75]$ & $0.71[0.68-0.74]$ & $0.59[0.56-0.62]$ & $0.62[0.59-0.65]$ & $0.61[0.58-0.63]$ \\
\hline Schonberg & $0.72[0.70-0.74]$ & $0.64[0.61-0.67]$ & $0.58[0.55-0.61]$ & $0.58[0.55-0.61]$ & $0.62[0.59-0.65]$ \\
\hline Lee & $0.72[0.70-0.74]$ & $0.65[0.62-0.68]$ & $0.57[0.54-0.61]$ & $0.57[0.55-0.60]$ & $0.59[0.56-0.61]$ \\
\hline
\end{tabular}

Abbreviations: $A D L$, activities of daily living; IADL, instrumental activities of daily living

Models adjust for age and sex, and accounted for complex survey design. Samples for ADL and IADL disability exclude baseline maximal disability. Cognitive impairment defined by abnormal performance in any of orientation, memory, or executive function. Mobility impairment defined by inability to walk 6 blocks or climb a flight of stairs

percentile of frailty, Schonberg, and Lee indices, respectively) (Fig. 1b). The C-statistics (95\% CIs) were similar: frailty index, 0.78 (0.77-0.80); Schonberg index, 0.79 (0.78-0.81); and Lee index, $0.78(0.77-0.80)$ (Table 2$)$. Mortality was higher in subgroups with cognitive impairment $(29.2 \%)$ or mobility impairment (27.9\%). In these subgroups, mortality discrimination was similar among the three indices but lower relative to performance in the overall cohort. Sensitivity analysis conducted without adjustment for age and sex did not appreciably change results (Supplementary Table 4).]->

\section{Predicting Decline in ADL Disability at 1 Year}

At 1 year, 304 (3.9\%) died, and 1103 (16.5\%) had incomplete follow-up. Those who were excluded from secondary analyses were older, frailer, and had a higher prevalence of baseline ADL disability (Supplementary Table 5). Of the 5626 participants remaining at 1 year, 79 were excluded due to maximal ADL disability at baseline. Of the remaining 5547 participants, $747(10.5 \%)$ experienced decline in ADL function (Table 2). The greatest difference across risk categories was observed for frailty index ( $<10 \%$ vs $\geq 90 \%$ percentile scores: frailty index, $1.2 \%$ vs $41.6 \%$; Schonberg index, $2.7 \%$ vs
29.4\%; Lee index, $3.3 \%$ vs $33.1 \%$ ) (Fig. 1c). The frailty index had higher C-statistics (95\% CI) compared with Schonberg and Lee indices in the total population (frailty index, 0.80 [0.78-0.83]; Schonberg index, 0.74 [0.72-0.76]; Lee index, $0.74[0.71-0.77])$. The results were similar in all subgroups. Although C-statistics were among those with mobility impairment, frailty index had a higher C-statistic $(0.71$ [0.68-0.74]) than Schonberg index $(0.64$ [0.61-0.67]) and Lee index $(0.65$ $[0.62-0.68])$.

\section{Predicting Decline in IADL Function at 1 Year}

Among the 5626 participants with follow-up data at 1 year, 402 were excluded due to maximal IADL disability at baseline. Among the remaining 5224 participants, 1402 (24.6\%) had decline in IADLs at 1 year (Table 2). Incidence was highest in participants with cognitive impairment (34.4\%) and mobility impairment (32.8\%) at baseline, with similar distribution of incidence across percentiles by risk index (Fig. 1d). C-statistics were similar between frailty index (0.61 [0.59-0.63]), Schonberg index (0.60 [0.59-0.62]), and Lee index $(0.60$ [0.58-0.62]) in the overall population and all subgroups. 


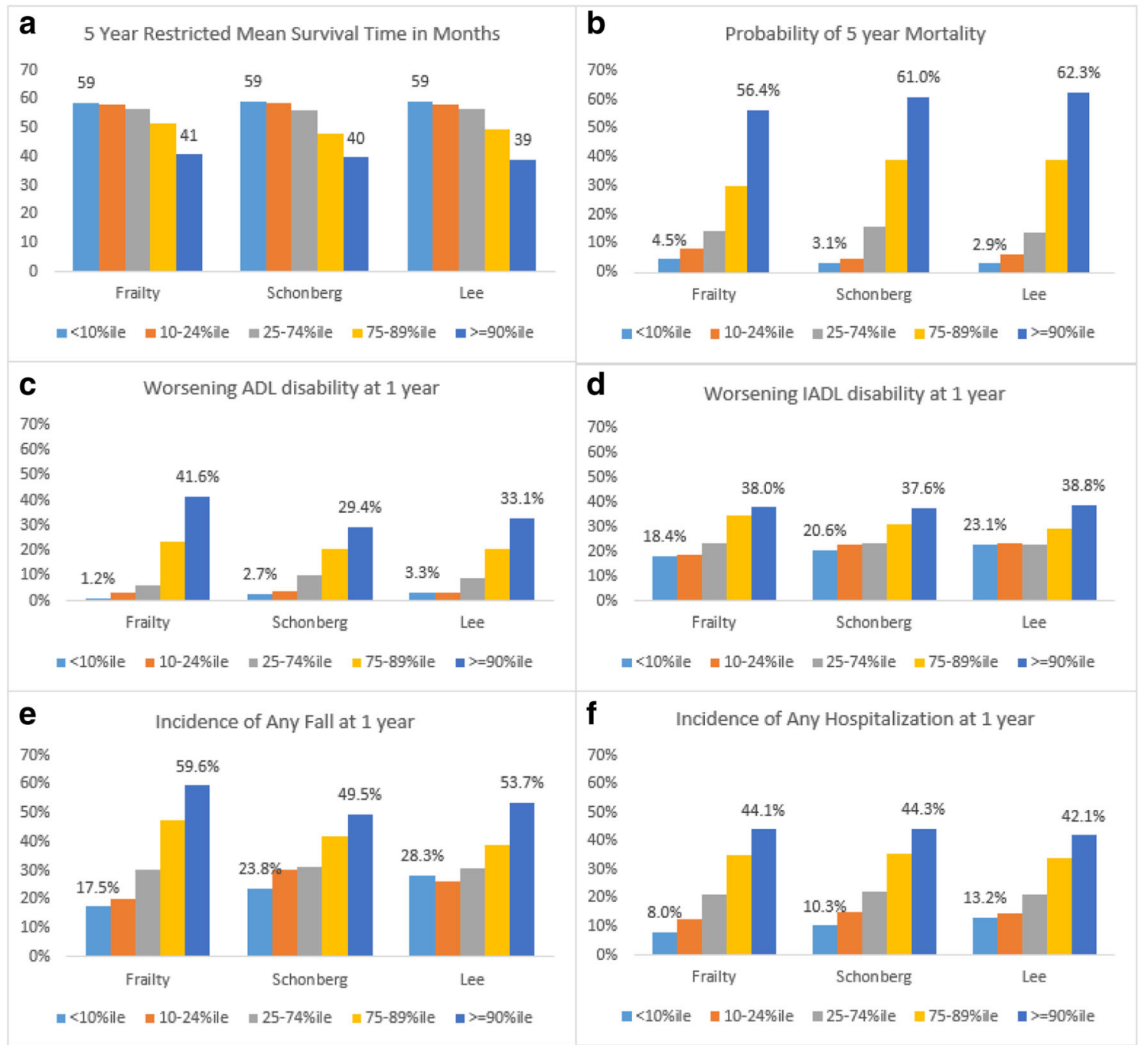

Fig. 1 Comparison between risk percentile categories of the Comprehensive Geriatric Assessment-based Frailty Index, Schonberg Index, and Lee Index. The 10th, 25th, 75th, and 90th percentile cutoffs for indices are as follows: frailty index $(0.06,0.09,0.25,0.40)$, Schonberg index $(3,5$, $11,14)$, and Lee index $(4,5,10,13)$. For all indices, higher risk categories are worse. Panel a compares 5 -year restricted mean survival time in months. Panel b shows the Kaplan-Meier estimator of 5-year mortality risk for each risk category for each index. Panels $c$ through $f$ compare the incidence of adverse outcomes each risk category for each index. For all indices, risk increases with higher scores. The frailty index had statistically significantly improved discrimination of falls and decline in ADL function.

\section{Prediction of Fall at 1 Year}

A total of $1881(32.6 \%)$ of 5626 participants had at least one fall in the year following the baseline assessment. The frailty index had the greatest difference in incidence across risk categories $(<$ $10 \%$ vs $\geq 90 \%$ percentile, $17.5 \%$ vs $59.6 \%$ ), compared with Schonberg index $(23.8 \%$ vs $49.5 \%)$ or Lee index $(28.3 \%$ vs $53.7 \%$ ) (Fig. 1e). The C-statistic was higher for frailty index (0.66 [0.65-0.68]) than Schonberg (0.61 [0.58-0.63]) and Lee (0.61 [0.59-0.63]) indices. While the highest risk of fall was observed in the group with mobility impairment (46.4\%), Cstatistics were lowest in this subgroup for all three indices (frailty index, 0.62 [0.59-0.65]; Schonberg index, 0.58 [0.55$0.61]$; Lee index, 0.57 [0.55-0.60]) (Table 2).

\section{Prediction of Hospitalization at 1 Year}

During the 1-year follow-up, 1397 (22.5\%) of 5626 participants were hospitalized. Increasing risk categories in all indices had higher rates of hospitalizations $(<10 \%$ vs $\geq 90 \%$ percentile scores: frailty index, $8.0 \%$ vs $44.1 \%$; Schonberg index, $10.3 \%$ vs $44.3 \%$; Lee index, $13.2 \%$ vs $42.1 \%$ ) (Fig. $1 f$ ). Both frailty index $(0.68[0.66-0.70])$ and Schonberg index (0.68 [0.66-0.69]) had similar C-statistics, while the Lee index had a C-statistic of 0.65 (0.63-0.67) (Table 2).

\section{DISCUSSION}

In this study, we compare frailty index with well-established mortality indices in a contemporary nationally representative sample of Medicare beneficiaries. For the first time, our results show comparable predictive ability of a frailty index to the Schonberg and Lee indices for mortality and superior ability for prediction of decline in ADLs and falls. Although predictive performance declined in beneficiaries with cognitive impairment and mobility impairment, the frailty index 
consistently performed as well as or better than the Schonberg and Lee indices across all subgroups.

Our results have important implications for the clinical management of medically complex older adults. Risk stratification is critical to personalized care for older adults, including individualizing treatment targets and counseling patients and families (e.g., hospice referral with estimated life expectancy $<6$ months). Although frailty index and both prognostic models predicted mortality well, traditional models incorporate demographic information, which is non-modifiable. By contrast, the frailty index contains multiple domains that are potentially modifiable, and lends itself to intervention. For example, if deficits are clustered in physical tasks, a clinician may be guided to focus on physical therapy and rehabilitation (Supplementary Figure 1). Recent work illustrates how a frailty index predicts chemotherapy outcomes in older patients, and may be used to individually tailor therapies. ${ }^{22}$ Thus, the frailty index acts beyond a predictive role, and can directly inform clinical management of patients.

There was variation in discrimination of frailty, Schonberg, and Lee indices across different subgroups. All three indices had lower C-statistics in subgroups with cognitive impairment or mobility impairment, which had higher mortality and incidence of other adverse outcomes. Previous research has shown model discrimination tends to be lower in sicker populations, such as nursing home residents, ${ }^{23}$ highlighting the challenges of risk prediction in highly morbid populations. Since mortality remains a challenging outcome to predict, and patients may weigh other outcomes more highly, prediction of mortality remains necessary but insufficient for framing routine clinical conversations in high risk patients. Superior performance of frailty index for ADL disability and falls suggests that the frailty index may capture additional risk factors that are not well measured in the prognostic indices.

Lastly, while mortality risk is commonly used for prognostication, there are meaningful outcomes that impact quality of life, which may be equally important to many older patients. ${ }^{24}$, ${ }^{25}$ Thus, risk stratification based solely on mortality prediction may not align with patient preferences. Existing indices for other outcomes such as ADL disability or falls may require special tools, and have modest C-statistics (e.g., 0.64 for any fall ${ }^{26}$ and $0.65-0.70$ for ADL disability). ${ }^{27}$ Since the frailty index is a unifying predictor of multiple health outcomes, it may be useful to reframe shared decision-making.

There are concerns that frailty index calculation is impractical. The frailty index used for this study is based on routinely captured items in geriatric clinical assessment. ${ }^{28}$ While a comprehensive geriatric assessment of a new patient takes generally 45-60 min, ${ }^{29}$ many variables (e.g., comorbidities, ADLs) are available in routine practice. Although performance variables, such as gait speed or grip strength, may not be typically measured, the frailty index calculated without performance variables can be used. ${ }^{30}$ Frailty index calculation can be further assisted with online calculators (e.g., http://bit.ly/cgafi), and has been adapted to electronic medical records ${ }^{31}$ and claims data, ${ }^{32}$ potentially alleviating the burden of calculation from clinical healthcare providers.

Our study has limitations to note. Our results from older community-dwelling Medicare beneficiaries may not be generalizable to institutionalized or younger non-Medicare populations. We acknowledge that roughly a third of NHATS participants were lost to follow-up over 5 years. These participants were healthier at baseline with lower scores for all indices; thus, overall discrimination for all indices may be underestimated. Analysis of 1-year adverse outcomes was restricted to survivors (Supplemental Table 4); thus, risk of these events may have been underestimated. Because these patterns were consistent for all indices, we do not expect this biases our comparative results in favor of any specific index for any particular outcome. The Lee index was developed to predict 4-year mortality, however has been validated for 10year mortality as well. Since a frailty index is not developed for mortality prediction, we were unable to evaluate calibration of frailty index for mortality. Nonetheless, the observed risk of mortality among frail subgroups in our sample was comparable with the previously reported mortality risk in large population-based studies that applied a deficit-accumulation frailty index. ${ }^{33}$ Additionally, the estimated mortality for the Lee and Schonberg indices in our sample was comparable with prior literature. ${ }^{5,6,23}$ Finally, the observed differences in $\mathrm{C}$-statistics between indices may not necessarily indicate clinically meaningful differences.

In conclusion, a frailty index predicts mortality comparably with validated prognostic indices in community-dwelling older adults. Furthermore, it can predict risk of ADL disability and falls better than prognostic indices. Thus, a frailty index may offer a useful approach to risk assessment of complex patients with competing health priorities. Additional research is needed regarding how a frailty index can be best communicated and translated into clinical practice.

Corresponding Author: Sandra M. Shi, MD; Department of Medicine, Beth Israel Deaconess Medical Center, Harvard Medical School, Boston, MA, USA (e-mail: sandrashi@hsl.harvard.edu).

Author Contributions Study design and concept: SS, DK. Statistical analysis: SS, DK, EM. Interpretation of data: All authors. Drafting and revision of manuscript: All authors. All authors approved the final version of the manuscript.

Funding Information Dr. Shi receives sponsorship from the Harvard Translational Research in Aging Training Program, T32 AG023480. Dr. Mitchell is supported by NIH-NIA K24AG033640.

\section{Compliance with Ethical Standards:}

Conflict of Interest: The authors have no conflicts of interest to disclose.

\section{REFERENCES}

1. Ickowicz E. Guiding principles for the care of older adults with multimorbidity: An approach for clinicians: American Geriatrics Society 
expert panel on the care of older adults with multimorbidity. J Am Geriatr Soc 2012;60(10):1-25. https://doi.org/10.1111/j.1532-5415.2012. 04188.x

2. Tinetti ME, Naik AD, Dodson JA. Moving From Disease-Centered to Patient Goals - Directed Care for Patients With Multiple Chronic Conditions. JAMA Cardiol 2016;1:9-10. https://doi.org/10.1001/ jamacardio.2015.0248. Conflict

3. Gill TM. The central role of prognosis in clinical decision making. J Am Med Assoc 2012;307(2):199-200. https://doi.org/10.1001/jama.2011. 1992.THE

4. Tinetti ME, Esterson J, Ferris R, Posner P, Blaum CS. Patient PriorityDirected Decision Making and Care for Older Adults with Multiple Chronic Conditions. Clin Geriatr Med 2016;32(2):261-275. https://doi. org/10.1016/j.cger.2016.01.012

5. Schonberg MA, Davis RB, McCarthy EP, Marcantonio ER. Index to predict 5-year mortality of community-dwelling adults aged 65 and older using data from the national health interview survey. J Gen Intern Med 2009;24(10):1115-1122. https://doi.org/10.1007/s11606-009-1073-y

6. Lee SJ, Lindquist $\mathbf{K}$, Segal MR, Covinsky KE. Development and validation of a prognostic index for 4-year mortality in older adults. JAMA. 2006;295(7):801-808. https://doi.org/10.1001/jama.295.7.801

7. Bandeen-Roche $\mathbf{K}$, Seplaki CL, Huang $\mathbf{J}$, et al. Frailty in Older Adults: A Nationally Representative Profile in the United States. J Gerontol Ser A Biol Sci Med Sci 2015;70(11):1427-1434. https://doi.org/10.1093/ gerona/glv133

8. Fried LPP, Tangen CMM, Walston $\mathbf{J}$, et al. Frailty in older adults: evidence for a phenotype. J Gerontol A Biol Sci Med Sci 2001;56(3):M146-M156. https://doi.org/10.1093/gerona/56.3.M146

9. Rockwood K, Mitnitski A. Frailty in relation to the accumulation of deficits . J Gerontol A Biol Sci Med Sci . 2007;62(7):722-727

10. Song X, Mitnitski A, Rockwood K. Prevalence and 10-Year outcomes of frailty in older adults in relation to deficit accumulation. J Am Geriatr Soc 2010;58(4):681-687. https://doi.org/10.1111/j.1532-5415.2010.02764. $\mathrm{x}$

11. Rockwood K, Mitnitski A, Song X, Steen B, Skoog I. Long-term risks of death and institutionalization of elderly people in relation to deficit accumulation at age 70. J Am Geriatr Soc 2006;54(6):975-979. https://doi.org/10.1111/j.1532-5415.2006.00738.x

12. Blodgett J, Theou O, Kirkland S, Andreou P, Rockwood K. Frailty in NHANES: Comparing the frailty index and phenotype. Arch Gerontol Geriatr 2015;60(3):464-470. https://doi.org/10.1016/j.archger.2015. 01.016

13. Theou O, Brothers TD, Mitnitski A, Rockwood K. Operationalization of frailty using eight commonly used scales and comparison of their ability to predict all-cause mortality. J Am Geriatr Soc 2013;61(9):1537-1551. https://doi.org/10.1111/jgs.12420

14. Shi S, Afilalo J, Lipsitz LA, et al. Frailty Phenotype and Deficit Accumulation Frailty Index in Predicting Recovery After Transcatheter and Surgical Aortic Valve Replacement. J Gerontol Ser A. 2018;XX(Xx): 18. https://doi.org/10.1093/gerona/gly196

15. Kasper JD, Freedman VA. National Health and Aging Trends Study ( NHATS ) User Guide: Rounds 1, 2, 3, 4 \& 5 Beta Release. 2016. www. NHATS.org.

16. Jones DM, Song $\mathbf{X}$, Rockwood $\mathbf{K}$. Operationalizing a frailty index from a standardized comprehensive geriatric assessment . J Am Geriatr Soc . 2004;52(11): 1929-1933. https://doi.org/10.1111/j.1532-5415.2004. $52521 . x$

17. Montaquila J, Freedman V, Edwards B, Kasper J. National Health and Aging Trends Study Round 1 Sample Design and Selection. NHATS Technical Paper \# 1. 2012:1-8. Available at www.NHATS.org.
18. Simning A, Fox ML, Barnett SL, Sorensen S, Conwell Y. Depressive and Anxiety Symptoms in Older Adults With Auditory, Vision, and Dual Sensory Impairment. J Aging Health 2018:1-23. https://doi.org/10. 1177/0898264318781123

19. Kasper JD, Freedman VA, Spillman B. Classification of Persons by Dementia Status in the National Health and Aging Trends Study. Balt Johns Hopkins Univ Sch Public Heal Available www.NHATS.org. 2013;(July):14. https://doi.org/10.1007/s00129-002-1155-y

20. Kim DH, Uno H, Wei LJ. Restricted mean survival time as a measure to interpret clinical trial results. JAMA Cardiol 2017;2(11):1179-1180. https://doi.org/10.1001/jamacardio.2017.2922

21. Newson RB. Comparing the predictive powers of survival models using Harrell's C or Somers' D. Stata J 2010;10(3):339-358.

22. McCarthy AL, Peel NM, Gillespie KM, et al. Validation of a frailty index in older cancer patients with solid tumours. BMC Cancer 2018;18(1):1-8. https://doi.org/10.1186/s12885-018-4807-6

23. Yourman L, Lee SJ, Schonberg MA, Widera E, Smith AK. Prognostic Indices for Older Adults A Systematic Review. JAMA. 2012;307(2):182192.

24. Schoenborn NL, Janssen EM, Boyd C, et al. Older Adults' Preferences for Discussing Long-Term Life Expectancy: Results From a National Survey. Ann Fam Med 2018. https://doi.org/10.1370/afm.2309

25. Schoenborn NL, Boyd C, Cayea $\mathbf{D}$, et al. Incorporating prognosis in the care of older adults with multimorbidity: Description and evaluation of a novel curriculum. BMC Med Educ 2015. https://doi.org/10.1186/ s12909-015-0488-x

26. Palumbo P, Palmerini L, Bandinelli S, Chiari L. Fall risk assessment tools for elderly living in the community: Can we do better? PLoS One 2015;10(12):1-13. https://doi.org/10.1371/journal.pone.0146247

27. Kim DH, Newman AB, Lipsitz LA. Prediction of severe, persistent activity-of-daily-living disability in older adults. Am J Epidemiol 2013;178(7): 1085-1093. https://doi.org/10.1093/aje/kwt097

28. Jones D, Song X, Mitnitski A, Rockwood K. Evaluation of a frailty index based on a comprehensive geriatric assessment in a population based study of elderly Canadians . Aging Clin Exp Res . 2005;17(6):465-471. http://www.ncbi.nlm.nih.gov/pubmed/16485864.

29. Huisingh-Scheetz M, Martinchek M, Becker Y, Ferguson MK, Thompson K. Translating Frailty Research Into Clinical Practice: Insights From the Successful Aging and Frailty Evaluation Clinic. J Am Med Dir Assoc 2019;20(6):672-678. https://doi.org/10.1016/j.jamda.2018.12.002

30. Clegg A, Bates $\mathbf{C}$, Young $\mathbf{J}$, et al. Development and validation of an electronic frailty index using routine primary care electronic health record data. Age Ageing 2016;45(3):353-360. https://doi.org/10.1093/ageing/ afw039

31. Pajewski NM, Lenoir K, Wells BJ, Williamson JD, Callahan KE. Frailty Screening Using the Electronic Health Record Within a Medicare Accountable Care Organization. J Gerontol Ser A. 2019;XX(Xx):1-7. https://doi.org/10.1093/gerona/glz017

32. Kim DH, Schneeweiss S, Glynn RJ, Lipsitz LA, Rockwood K, Avorn J. Measuring Frailty in Medicare Data: Development and Validation of a Claims-Based Frailty Index. Journals Gerontol - Ser A Biol Sci Med Sci 2018;73(7):980-987. https://doi.org/10.1093/gerona/glx229

33. Rockwood $\mathbf{K}$, Song $\mathbf{X}$, MacKnight $\mathbf{C}$, et al. A global clinical measure of fitness and frailty in elderly people. CMAJ. 2005;173(5):489-495. https://doi.org/10.1503/cmaj.050051

Publisher's Note Springer Nature remains neutral with regard to jurisdictional claims in published maps and institutional affiliations. 